\title{
Robot-Assisted Radical Prostatectomy in Hong Kong: A Review of 235 Cases
}

\author{
Kam Hung Sidney Yip, MBBS,, ${ }^{1,2}$ Chi-hang Yee, MBBS, ${ }^{2}$ Chi-fai Ng, M.D.,, ${ }^{1,2}$ Nga-yee Lam, B.Sc., \\ Kwan-lun Ho, MBBS, ${ }^{3}$ Wai-kit Ma, M.B., Ch.B., ${ }^{4}$ Cheuk-man Li, M.B., Ch.B., ${ }^{5}$ See-ming Hou, MBBS, ${ }^{2}$ \\ Po-chor Tam, MBBS, ${ }^{3}$ Ming-kwong Yiu, MBBS, ${ }^{4}$ and Cho-wai Fan, MBBS ${ }^{5}$
}

\begin{abstract}
Objectives: To report the outcome of all robot-assisted laparoscopic radical prostatectomy (RALP) in the public health care system in Hong Kong.

Patients and Methods: All patients who underwent RALP in the public health care system with at least 1 year of follow-up were evaluated. Data analysis included age, body mass index, preoperative prostate-specific antigen (PSA) level, D'Amico risk category, operative details, pathologic stage, follow-up continence, potency, and biochemical recurrence.

Results: Between 2005 and 2009, 235 patients underwent RALP, with a mean age of $66.4 \pm 5.9$ years and a mean preoperative PSA level of $11.0 \pm 10.5 \mathrm{ng} / \mathrm{mL}$. Complications were $16(7 \%)$ in total. There were $176(74.9 \%)$ patients with $\mathrm{pT}_{2}$ disease and $55(23.4 \%)$ patients with $\mathrm{pT}_{3}$ disease. The overall rate of positive surgical margins (PSM) was $20.7 \%$. At postoperative 12 months, $72.5 \%$ of the patients were pad free. For those 83 preoperative potent patients having nerve-sparing surgery, the overall trifecta rate at 12 months was $37.3 \%$. Multivariate analysis identified that pathologic T staging was significantly associated with PSM, with an odds ratio (OR) of $7.884(95 \%$ confidence interval $[C I]: 3.576-17.379 ; P<0.001)$ for the $\mathrm{pT}_{3}$ group compared with the $\mathrm{pT}_{2}$ group. When comparing D'Amico medium- and high-risk categories with low-risk categories, they were found to be significantly associated with biochemical failure (medium- compared with low-risk: OR=3.536, 95\% CI: $1.253-$ 10.173, $P=0.016$; high- compared with low-risk: $\mathrm{OR}=10.214,95 \% \mathrm{CI}: 2.958-35.274, P<0.001$ ).

Conclusions: Our data demonstrate the feasibility, safety, and efficacy of RALP in low-to-intermediate volume centers. Our early oncologic outcomes were significantly correlated with pathologic stage and D'Amico risk stratification.
\end{abstract}

\section{Introduction}

$\mathbf{P}$ ROSTATE CANCER IS THE SECOND MOST COMMON CANCER in the world, with a world age-standardized rate of 28 per $100,000 .{ }^{1}$ While the Western nations have a higher incidence of prostate cancer, it is increasing rapidly in Asian countries because of a more westernized lifestyle. ${ }^{2}$ In Hong Kong, prostate cancer is currently the third most common cancer in men and ranks fifth in cancer mortality. ${ }^{3}$

For localized prostate cancer, radical prostatectomy (RP) remains the standard for long-term cure. ${ }^{4}$ In an effort to reduce morbidity of open RP, Schusessler and associates ${ }^{5}$ first described a minimally invasive surgical approach to treat patients with prostate cancer with laparoscopic RP in 1997. The subsequent introduction of the da Vinci ${ }^{\circledR}$ Surgical System
(Intuitive Surgical, Sunnyvale, CA) paved the breakthrough in minimally invasive prostatectomy. With the promise of improved ergonomics and a shortened learning curve that accompany the da Vinci Surgical System, robot-assisted laparoscopic radical prostatectomy (RALP) is rapidly gaining acceptance in the urologic community. ${ }^{6}$

RALP is blossoming in Asia as well as in the West. Since the implementation of robotics in the Asian region, a dramatic increase in the number of RALP has been seen. ${ }^{7}$ This "halo effect," as referred to by Sung and colleagues, ${ }^{8}$ contributes to the evolution of robotic surgical techniques into maturity in the region. Hong Kong had its first robotic system installed in $2005^{9}$ and is among the first few cities in Asia that acquired such technology. While most of the RALP in the West are performed in the context of a private insurance scheme, the

\footnotetext{
${ }^{1}$ Department of Surgery, Chinese University of Hong Kong, Hong Kong.

${ }^{2}$ Department of Surgery, Prince of Wales Hospital, Hong Kong.

${ }^{3}$ Department of Surgery, Queen Mary Hospital, Hong Kong.

${ }^{4}$ Department of Surgery, Princess Margaret Hospital, Hong Kong.

${ }^{5}$ Department of Surgery, Pamela Youde Nethersole Eastern Hospital, Hong Kong.
} 
majority of robot-assisted prostatectomies in Hong Kong are performed in the public sector. We report the outcome of all RALP in the public health care system in Hong Kong since its launch in 2005.

\section{Patients and Methods}

There are a total of five da Vinci Surgical Systems for clinical use in Hong Kong. Four of them are being used in the public sector in four different hospitals. They were introduced between 2005 and 2009. We evaluated all patients who underwent RALP in the public health care system with at least 1 year of follow-up.

All surgeons who were involved in the series were proficient in laparoscopic surgery, and some had previous robotic experience in other localities. The majority of cases were performed using the six-port technique. All adopted the transperitoneal approach. The general criteria for considering a nerve-sparing procedure were potent patients in the low to moderate D'Amico risk category. The final decision also involved patient preference and the intraoperative condition, Most of the nerve-sparing procedures were performed with the interfascial technique. A few cases were performed intrafascially. The vesicourethral anastomosis was created using continuous running suture as originally described by van Velthoven and coworkers. ${ }^{10}$

Data collection included age, body mass index (BMI), preoperative prostate-specific antigen (PSA) level, D'Amico risk category, ${ }^{1}$ operative details, postoperative course, pathologic stage, follow-up continence, potency, and biochemical recurrence. Follow-up protocols across the four centers were similar. Clinical outcome of patients at postoperative 3, 6, and 12 months were reviewed. Continence status was classified into four groups: (1) Pad free, (2) use of one pad for security or occasional stress incontinence, (3) two to three pads per day, and (4) four or more pads per day. Potency was defined as the ability to achieve and maintain satisfactory erections firm enough for sexual intercourse for more than $50 \%$ of times, with or without the use of oral phosphodiesterase type 5 inhibitors. Biochemical recurrence was defined as two consecutive values of PSA $>0.2 \mathrm{ng} / \mathrm{mL}$.

Descriptive statistics were used to characterize the clinical characteristics of the study cohort. Univariate and multivariate logistic regression analyses were performed to identify clinical covariates that were significantly associated with surgical margin status, erectile function status, continence status, and biochemical recurrence. Odds ratios (ORs) and their associated $95 \%$ confidence interval (CI) were estimated. $P$ values $<0.05$ were considered statistically significant. SPSS software package version 17 (SPSS Inc, Chicago, IL) was used for all calculations.

\section{Results}

Between November 2005 and December 2009, 235 consecutive patients underwent RALP in the public sector in Hong Kong. Patient characteristics are outlined in Table 1. Median follow-up time was 20 months. The majority of the cohort belonged to the $\mathrm{D}^{\prime}$ Amico low-risk category (65\%). About half of the patients were potent preoperatively.

Table 1 summarizes the perioperative parameters. Among the 235 cases, 64 cases underwent pelvic lymphadenectomy. Mean operative time was $362 \pm 124$ mins. Mean estimated
Table 1. Patient Characteristics and Perioperative Parameters

\begin{tabular}{|c|c|}
\hline Parameter & Value \\
\hline Patients (n) & 235 \\
\hline Mean age, $\mathrm{y} \pm \mathrm{SD}$ & $66.4 \pm 5.9$ \\
\hline Mean BMI, $\mathrm{kg} / \mathrm{m}^{2} \pm \mathrm{SD}$ & $24.8 \pm 3.2$ \\
\hline Mean PSA, ng/mL $\pm S D$ & $11.0 \pm 10.5$ \\
\hline \multicolumn{2}{|l|}{ Clinical stage } \\
\hline $\mathrm{cT}_{1 \mathrm{a}}(\mathrm{n})$ & $1(0.4 \%)$ \\
\hline $\mathrm{cT}_{1 \mathrm{~b}}(\mathrm{n})$ & $4(1.7 \%)$ \\
\hline $\mathrm{cT}_{1 \mathrm{c}}(\mathrm{n})$ & $175(74.5 \%)$ \\
\hline $\mathrm{cT}_{2 \mathrm{a}}(\mathrm{n})$ & $39(16.6 \%)$ \\
\hline $\mathrm{cT}_{2 \mathrm{~b}}(\mathrm{n})$ & $8(3.4 \%)$ \\
\hline $\mathrm{cT}_{2 \mathrm{c}}(\mathrm{n})$ & $8(3.4 \%)$ \\
\hline \multicolumn{2}{|l|}{ Preoperative total Gleason score } \\
\hline $6(n)$ & $172(73.2 \%)$ \\
\hline $7(\mathrm{n})$ & $40(17.0 \%)$ \\
\hline $8(\mathrm{n})$ & $14(6.0 \%)$ \\
\hline $9(\mathrm{n})$ & $4(1.7 \%)$ \\
\hline \multicolumn{2}{|l|}{$\mathrm{D}^{\prime}$ Amico risk category } \\
\hline Low $(\mathrm{n})$ & $152(65 \%)$ \\
\hline Medium (n) & $62(26 \%)$ \\
\hline High $(\mathrm{n})$ & $20(9 \%)$ \\
\hline Preoperative potent patients (n) & $132(56 \%)$ \\
\hline Mean follow-up duration, mos $\pm S D$ & $24 \pm 12$ \\
\hline Mean operative time, $\min \pm S D$ & $362 \pm 124$ \\
\hline Mean blood loss, $\mathrm{mL} \pm \mathrm{SD}$ & $626 \pm 657$ \\
\hline Conversion to open (n) & $4(1.7 \%)$ \\
\hline Mean catheter in-situ time, $\mathrm{d} \pm \mathrm{SD}$ & $13 \pm 6$ \\
\hline Mean hospital stay, $\mathrm{d} \pm \mathrm{SD}$ & $7 \pm 4$ \\
\hline \multicolumn{2}{|l|}{ Complications } \\
\hline Anastomotic leakage (n) & $6(2.6 \%)$ \\
\hline Deep vein thrombosis (n) & $3(1.3 \%)$ \\
\hline Wound infection (n) & $3(1.3 \%)$ \\
\hline Rectal perforation (n) & $1(0.4 \%)$ \\
\hline Pulmonary embolism (n) & $1(0.4 \%)$ \\
\hline Urethral stricture (n) & $1(0.4 \%)$ \\
\hline Lymphocele (n) & $1(0.4 \%)$ \\
\hline Mortality & $1(0.4 \%)$ \\
\hline
\end{tabular}

$\mathrm{SD}=$ standard deviation; $\mathrm{BMI}=$ body mass index; $\mathrm{PSA}=$ prostatespecific antigen.

blood loss was $626 \pm 657 \mathrm{~mL}$. Four patients needed open conversion in our series; two of the procedures were converted for hemostasis purposes, and the other two because of the dense adhesion encountered during dissection. Mean hospital stay and mean catheter time were $7 \pm 4$ days and $13 \pm 6$ days, respectively.

Complications were $16(7 \%)$ in total and comprised 6 anastomotic leakages (2.6\%), 3 deep vein thrombosis (DVT) (1.3\%), 3 wound infection (1.3\%), 1 rectal perforation $(0.4 \%), 1$ urethral stricture $(0.4 \%), 1$ lymphocele $(0.4 \%)$, and 1 pulmonary embolism $(0.4 \%)$. The patient with pulmonary embolism was about to be discharged when a sudden postoperative event developed on postoperative day 9 and he succumbed.

Histopathologic analysis of the RALP specimen found a mean prostate weight of $49.4 \pm 20.8 \mathrm{~g}$ (Table 2). There were 176 $(74.9 \%)$ patients with $\mathrm{pT}_{2}$ disease while $55(23.4 \%)$ patients had $\mathrm{pT}_{3}$ disease. The overall rate of positive surgical margin (PSM) was $20.7 \%$. In the subcategorical analysis review, $\mathrm{pT}_{2}$ patients and $\mathrm{pT}_{3}$ patients had a PSM rate of $12 \%$ and $41 \%$, respectively. At postoperative 12 months, $72.5 \%$ of the patients were pad-free (Table 3). For those 83 preoperative 
Table 2. Oncologic Outcome

\begin{tabular}{lc}
\hline Parameter & Value \\
\hline Mean prostate specimen weight, $\mathrm{g} \pm \mathrm{SD}$ & $49.4 \pm 20.8$ \\
Pathological T stage $(\mathrm{n})$ & $176(74.9 \%)$ \\
$\mathrm{T}_{2}(\mathrm{n})$ & $55(23.4)$ \\
$\mathrm{T}_{3}(\mathrm{n})$ & $4(1.7 \%)$ \\
$\mathrm{T}_{\times}(\mathrm{n})$ & \\
Prostate specimen Gleason score & $144(61.3 \%)$ \\
$6(\mathrm{n})$ & $63(26.8 \%)$ \\
$7(\mathrm{n})$ & $15(6.4 \%)$ \\
$8(\mathrm{n})$ & $6(2.6 \%)$ \\
$9(\mathrm{n})$ & $47(20.7 \%)$ \\
Positive surgical margin (n) & 21 \\
$\mathrm{~T}_{2}(\mathrm{n})$ & 26 \\
$\mathrm{~T}_{3}(\mathrm{n})$ & $22(9.4 \%)$ \\
Overall biochemical recurrence (n) & 7 \\
$\mathrm{D}^{\prime}$ Amico low risk (n) & 9 \\
$\mathrm{D}^{\prime}$ Amico medium risk (n) & 6 \\
$\mathrm{D}^{\prime}$ Amico high risk (n) & $18 \pm 11$ \\
Mean biochemical recurrence time, & \\
months \pm SD & \\
\hline
\end{tabular}

$\mathrm{SD}=$ standard deviation

potent patients who underwent nerve-sparing surgery (including unilateral nerve-sparing RALP), 31 (37.3\%) patients remained potent at postoperative 12 months (Table 4). In the cohort of these 83 patients, the overall trifecta rate (pad free, potent, no biochemical recurrence) at 12 months was $37.3 \%$.

Among the 235 cases, 29 (12.3\%) patients were given adjuvant therapies. These adjuvant therapies included radiotherapy $(n=18)$, antiandrogen $(n=1)$, surgical $(n=4)$, and medical castration $(n=6)$.

While no correlation was found between nerve sparing and PSM, multivariate analysis with logistic regression identified that pathologic $\mathrm{T}$ staging was significantly associated with PSM, with an OR of 7.884 (95\% CI: 3.576-17.379; $P<0.001)$ for $\mathrm{pT}_{3}$ group compared with the $\mathrm{pT}_{2}$ group (Table 5). There was a negative correlation between prostate specimen weight and PSM, with an OR of 0.972 (95\%CI: 0.950-0.994; $P=0.013$ ). D'Amico risk stratification had an implication on biochemical recurrence. When comparing medium- and high-risk categories with low-risk categories, they were found to be significantly associated with biochemical failure (mediumcompared with low-risk: $\mathrm{OR}=3.536,95 \% \mathrm{CI}$ : 1.253-10.173, $P=0.016$; high- compared with low-risk: $\mathrm{OR}=10.214,95 \% \mathrm{CI}$ : 2.958-35.274, $P<0.001)$.

\section{Discussion}

Since the first robot-assisted laparoscopic radical prostatectomy performed by Binder and Kramer ${ }^{12}$ in May 2000,

Table 3. Continence Outcome

\begin{tabular}{lccc}
\hline & 3 mos & 6 mos & 12 mos \\
\hline Pad-free (n) & $98(42.4 \%)$ & $138(60.8 \%)$ & $166(72.5 \%)$ \\
Occasional & $60(26.0 \%)$ & $35(15.4 \%)$ & $22(9.6 \%)$ \\
$\quad$ incontinence (n) & & & \\
2-3 pads per day (n) & $43(18.6 \%)$ & $35(15.4 \%)$ & $24(10.5 \%)$ \\
$\begin{array}{l}4 \text { or more pads } \\
\quad \text { per day (n) }\end{array}$ & $30(13.0 \%)$ & $19(8.4 \%)$ & $17(7.4 \%)$ \\
\hline
\end{tabular}

Table 4. Erectile Function Outcome of Preoperative Potent Patients

\begin{tabular}{lc}
\hline Parameter & Value \\
\hline $\begin{array}{l}\text { Preoperative potent patients undergoing } \\
\text { nerve-sparing RALP (n) }\end{array}$ & 83 \\
Postoperative 1-year potent patients (n) & $31(37.3 \%)$ \\
Number of patients on PDE5 inhibitor (n) & $45(54.2 \%)$ \\
\hline
\end{tabular}

RALP $=$ robot-assisted laparoscopic prostatectomy; PDE5=phosphodiesterase type.

RALP has become a popular approach for the management of prostate cancer in many regions. While the da Vinci robotic system offers several advantages for surgeons, including 10x magnification with three-dimensional stereoscopic optics and end-of-wrist instrument with 7 degrees of freedom in range of motion, its expensive implementation cost has been a concern to many centers. ${ }^{13}$ Contrary to other regions, the four robotic surgery systems in the public sector in Hong Kong were donated initially as charity and run subsequently by receiving support from the public health care system. Robotic surgery in Hong Kong thus carries a low price tag for patients, although not necessarily for the whole health care system.

Oncologic outcome would definitely be one of the main interests during the evaluation of any cancer surgery. The parameters for such assessment include overall survival, disease-specific survival, and biochemical recurrence-free survival, which is commonly defined as a PSA level $<0.2 \mathrm{ng} /$ $\mathrm{mL}$. Not all of these parameters are applicable, however, in the comparison between an RALP series and an open RP series at this junction, where the latter had 15-year outcome data available. ${ }^{14}$ Biochemical recurrence and surgical margin status are thus often used as surrogates for cancer control when evaluating oncologic efficacy.

The largest series of RALP to date was reported by Badani and colleagues ${ }^{15}$ from the Henry Ford Center in Detroit. It involved 2766 patients with a follow-up of up to 5 years. In their series, they reported a PSA recurrence of 7.27\%. Among $\mathrm{pT}_{2}$ and $\mathrm{pT}_{3}$ patients, the rates of $\mathrm{PSM}$ were $13 \%$ and $35 \%$, respectively. Patel and associates ${ }^{16}$ evaluated a singlesurgeon experience of 1500 consecutive RALP, and reported a PSM rate of $4 \%$ for $\mathrm{pT}_{2}$ and $34 \%$ for $\mathrm{pT}_{3}$.

Our series had a PSM rate of $12 \%$ for $\mathrm{pT}_{2}$ and $41 \%$ for $\mathrm{pT}_{3}$, and an overall PSM rate of $20.7 \%$. These figures fell within the range of previous reports in the literature, which showed a mean overall PSM rate of $15.2 \%$ (range of means $9.3 \%-33 \%$ ), a mean PSM rate for $\mathrm{pT}_{2}$ of $9.6 \%$ (range of means $2.5 \%-18 \%$ ), and a mean $\mathrm{PSM}$ rate for $\mathrm{pT}_{3}$ of $37.1 \%$ (range of means $20.9 \%$ $53.8 \%)^{17}$

A higher $\mathrm{T}$ stage of the tumor has been implicated to have a higher risk for PSM in the open RP series. ${ }^{18}$ There were very few reports in the literature on this subject for RALP, however. Weizer and coworkers ${ }^{19}$ reviewed a cohort of 633 patients, consisting of both open prostatectomy and RALP. It showed that patients with a lower pathologic stage $\left(\mathrm{pT}_{2}\right)$ had a lower risk of PSM compared with patients with $\mathrm{pT}_{3}$ or more disease. In a study by Ham and associates, ${ }^{20}$ the authors noted that there was a significant difference in PSM rate between clinically localized and locally advanced prostate cancer ( $48 \%$ vs 59\% respectively, $P<0.001$ ). Our finding of pathologic T stage being significantly associated with PSM rate in RALP would complement the 
Table 5. Multivariate Analysis of Perioperative Parameters on Positive Surgical Margins and Biochemical Recurrence

\begin{tabular}{|c|c|c|c|c|}
\hline & Factors & OR & $95 \% \mathrm{CI}$ & P value \\
\hline \multirow[t]{3}{*}{ Positive surgical margins } & $\mathrm{T}$ stage & 7.884 & $3.576,17.379$ & $<0.001$ \\
\hline & Operative time & 1.004 & $1.001,1.008$ & 0.012 \\
\hline & Prostate specimen weight & 0.972 & $0.950,0.994$ & 0.013 \\
\hline \multirow[t]{3}{*}{ Biochemical recurrence } & D'Amico low risk & 1.000(ref) & & \\
\hline & $\mathrm{D}^{\prime}$ Amico medium risk & 3.536 & $1.253,10.173$ & 0.016 \\
\hline & D'Amico high category & 10.214 & $2.958,35.274$ & $<0.001$ \\
\hline
\end{tabular}

$\mathrm{OR}=$ odds ratio; $\mathrm{CI}=$ confidence interval.

current reports in the literature. Furthermore, we demonstrated that D'Amico risk stratification was associated with biochemical failure in our cohort. The low-risk group was significantly better than those of medium- and high-risk groups. While this supports the D'Amico risk stratification as an effective predictor of early outcomes after RALP, our study is among the first few to evaluate the significance of risk stratification on early outcomes in a robotic cohort.

Besides complete removal of the cancer, recovery of potency and urinary continence are the other two long-term goals of RP. Potency is not easy to evaluate because there is significant variation in the literature as to the definition of potency after RP. A systemic review reported that at 12-month follow-up, potency rates ranged from $20 \%$ to $97 \% .{ }^{21}$ Our series reported a 12 -month potency rate of $37.3 \%$. While a lack of universal potency definition may render it difficult to compare our data with other cohorts, a few factors may account for our relatively low potency rate. Mulhall and colleagues ${ }^{22}$ demonstrated that postoperative early penile rehabilitation significantly increased the likeliness of achieving functional erections. The investigation by Rogers and coworkers $^{23}$ found that younger men had earlier return of sexual function and higher overall potency rates at 1 year after RALP. In our locality, an early penile rehabilitation program is not yet prevalent. In addition, when compared with many contemporary RALP series, which had a mean or median age range from 56.3 to 63.2 years, the mean age of our patients was older. ${ }^{17}$ These characteristics may have an influence on our potency results. Concerning urinary continence, our series demonstrated that 12 months after RALP, 80.1\% of the patients needed no absorbent pads or the use of 1 pad only for security. This result is in line with many other RALP series, which reported a continence rate of $70 \%$ to $97 \%$ at 12 months of follow-up. ${ }^{17}$

There was one mortality in our robot-assisted prostatectomy cohort. Pulmonary embolism (PE) developed in the patient on postoperative day 9. Postmortem examination did not reveal any DVT. In a multi-institutional study, the incidence of venous thromboembolism among 5951 patients who were treated with laparoscopic RP, with or without robot assistance, was examined. ${ }^{24}$ Symptomatic DVT or PE developed in 31 patients (0.5\%). Among these patients, four had PE without identified DVT. In their cohort, two patients died of PE. While this study focused on symptomatic thromboembolic events, Yee and colleagues $^{25}$ reported the findings of silent thromboembolic events. In that series, routine bilateral lower limb Doppler ultrasonography was performed for consecutive 65 patients after RALP on postoperative day 5. None of the patients had symptomatic DVT, but $1(3.1 \%)$ patient was found to have above-knee venous thromobosis and seven (21.9\%) patients were found to have below-knee venous thrombosis on ultra- sonographic examination. Both of these studies concluded that the incidence of symptomatic thromboembolic events was low after minimally invasive RP, and disease stage and pelvic lymph node dissection were not significant factors associated with thromboembolic events. Furthermore, data from the multi-institutional study do not support the administration of prophylactic heparin to all patients undergoing RP, in view of its minimal benefit with an increased complication rate. Based on current evidence, such judicious use of heparin is being adopted by the authors of this article.

The concurrent assessment of cancer-free, continence, and potency was termed "trifecta," after Salomon and coworkers $^{26}$ first reported their functional and oncologic outcomes combined in their series of open, laparoscopic, and perineal prostatectomy. There were very scarce reports of trifecta outcome in RALP patients in the literature. Such an account was first made by Shikanov and associates, ${ }^{27}$ who reported a trifecta rate of $44 \%$ at 12 months after RALP. In our series, the trifecta rate is $37.3 \%$ at 12 months. As previously illustrated, study designs and criteria for continence and potency often lack consistency. This may prevent us from directly comparing our results with other larger series-for example, Patel and colleagues, ${ }^{28}$ who achieved a trifecta rate of $86 \%$ at 12 months. Furthermore, the larger RALP series in the literature were often the report of a few high-volume surgeons. ${ }^{15,27,28}$ While some suggested that the learning curve was adequately developed after 25 to 45 cases of RALP, $, 6,16,29$ there is evidence that implied that the learning curve issue is much more complex, and individual surgeon results continue to improve up to 100 cases and beyond. ${ }^{16,30}$ Because only one center had performed more than 100 cases of RALP in our series, an accurate and comprehensive observation on the matter of learning curve in our series may be difficult.

When we reviewed the operative time, all except one center had a decrease in mean operative time when comparing the initial $50 \%$ of the cases with the later $50 \%$. This may serve as an indicator of showing a gradual mastering of the technique on the way and may imply being able to overcome the learning curve when cases accumulate. Although the implementation of the da Vinic robotic system is gaining popularity rapidly, most urologists performed much fewer prostatectomies than those high-volume surgeons quoted in the literature. ${ }^{31}$ Thus, our account of RALP may be more applicable to most institutions, which belong to the category of low-to-moderate volume center.

While the cost of robotic surgery is one of the issues that concerns health institutes, surgeons, and patients, we have not explored this aspect in the current study. In terms of cost analysis, there are two aspects to be considered. On the one 
hand, there are the additional implementation cost, maintenance cost, consumables cost, and maybe a longer operative time in certain aspects. These factors have also been elaborated by Barbash and associates. ${ }^{32}$ On the other hand, the key attributes of robotic surgery are increased precision, miniaturization, articulation beyond normal manipulation, and three-dimensional magnification. These would contribute to a reduction in the length of hospital stay, postoperative complications, blood loss, and time off work. When these improvements are accounted for, there may be a considerable savings to hospitals and society as a whole, offsetting the additional cost. Because of the complexity of the model, this may be beyond the scope of the current study, and we would hope that a separate investigation could be carried out on the subject of RALP cost analysis.

There are certain limitations of the present study. First, being a retrospective study, it was difficult to have a standardized follow-up protocol and surgical technique. Different surgeons in the cohort performed RALP with slightly different modifications. Such heterogeneity in technique may cloud the picture. In addition, the assessment of potency and urinary continence was made by patient-surgeon interviews. This may not correlate with patient self-assessments of health-related quality of life. Furthermore, the mean follow-up was relatively short when compared with the open prostatectomy series. This may affect trifecta outcomes given that with time, the biochemical recurrence rate increases, while continence and potency may improve. As the robotic technique becomes more established, we hope that longer-term data would be available.

\section{Conclusions}

We report a $37.3 \%$ rate of trifecta at 12 months after RALP in 235 patients. Our early oncologic outcomes were significantly correlated with pathologic stage and D'Amico risk stratification and appear to be similar to those in larger RALP series. Our data demonstrate the feasibility, safety, and efficacy of RALP in low-to-intermediate volume centers.

\section{Disclosure Statement}

No competing financial interests exist.

\section{References}

1. Ferlay J, Shin HR, Bray F, et al. GLOBOCAN 2008, Cancer Incidence and Mortality Worldwide: IARC CancerBase No. 10 [Internet]. Lyon, France: International Agency for Research on Cancer; 2010. Available from: http://globocan .iarc.fr. Accessed December 6, 2011.

2. Yip SKh, Sim HG. Robotic radical prostatectomy in east Asia: development, surgical results and challenges. Curr Opin Urol 2010;20:80-85.

3. Hong Kong Hospital Authority. Hong Kong Cancer Registry. hAccessed February 2011.

4. Bill-Axelson A, Holmberg L, Ruutu M, et al; Scandinavian Prostate Cancer Group Study No. 4. Radical prostatectomy versus watchful waiting in early prostate cancer. N Engl J Med 2005;352:1977-1984.

5. Schuessler WW, Schulam PG, Clayman RV, Kavoussi LR. Laparoscopic radical prostatectomy: Initial short-term experience. Urology 1997;50:854-857.

6. Ou YC, Yang CR, Wang J, et al. Robotic-assisted laparoscopic radical prostatectomy: Cearning curve of first 100 cases. Int J Urol 2010;17:635-640.
7. Sundram M. Asian robotic experience. Urol Oncol 2010;28:677681.

8. Sung ER, Jeong W, Park SY, et al. The "halo effect" in Korea: Change in practice patterns since the introduction of robotassisted laparoscopic radical prostatectomy. J Robotic Surg 2009;3:57-60.

9. Lo KL, $\mathrm{Ng} \mathrm{CF}$, Lam CN, et al. Short-term outcome of patients with robot-assisted versus open radical prostatectomy for localised carcinoma of prostate. Hong Kong Med J 2010;16:31-35.

10. Van Velthoven RF, Ahlering TE, Peltier A, et al. Technique for laparoscopic running urethrovesical anastomosis: The single knot method. Urology 2003;61:699-702.

11. D'Amico AV, Whittington R, Malkowicz SB, et al. Biochemical outcome after radical prostatectomy, external beam radiation therapy, or interstitial radiation therapy for clinically localized prostate cancer. JAMA 1998;280: 969-974.

12. Binder J, Kramer W. Robotically-assisted laparoscopic radical prostatectomy. BJU Int 2001;87:408-410.

13. Lotan Y, Bolenz C, Gupta A, et al. The effect of the approach to radical prostatectomy on the profitability of hospitals and surgeons. BJU Int 2010;105:1531-1535.

14. Han M, Partin AW, Pound CR, et al. Long-term biochemical disease-free and cancer-specific survival following anatomic radical retropubic prostatectomy. The 15-year Johns Hopkins experience. Urol Clin North Am 2001;28:555-565.

15. Badani KK, Kaul S, Menon M. Evolution of robotic radical prostatectomy: assessment after 2766 procedures. Cancer 2007;110:1951-1958.

16. Patel VR, Palmer KJ, Coughlin G, Samavedi S. Robotassisted laparoscopic radical prostatectomy: Perioperative outcomes of 1500 cases. J Endourol 2008;22:2299-2305.

17. Coelho RF, Chauhan S, Palmer KJ, et al. Robotic-assisted radical prostatectomy: A review of current outcomes. BJU Int 2009;104:1428-1435.

18. Boccon-Gibod L, Bertaccini A, Bono AV, et al. Management of locally advanced prostate cancer: A European consensus. Int J Clin Pract 2003;57:187-194.

19. Weizer AZ, Strope S, Wood DP Jr. Margin control in robotic and laparoscopic prostatectomy: Ahat are the REAL outcomes? Urol Oncol 2010;28:210-214.

20. Ham WS, Park SY, Rha KH, et al. Robotic radical prostatectomy for patients with locally advanced prostate cancer is feasible: Results of a single-institution study. J Laparoendosc Adv Surg Tech A 2009;19:329-332.

21. Ficarra V, Cavalleri S, Novara G, et al. Evidence from robotassisted laparoscopic radical prostatectomy: A systematic review. Eur Urol 2007;51:45-56.

22. Mulhall JP, Parker M, Waters BW, Flanigan R. The timing of penile rehabilitation after bilateral nerve-sparing radical prostatectomy affects the recovery of erectile function. BJU Int 2010;105:37-41.

23. Rogers CG, Su LM, Link RE, et al. Age stratified functional outcomes after laparoscopic radical prostatectomy. J Urol 2006;176:2448-2452.

24. Secin FP, Jiborn T, Bjartell AS, et al. Multi-institutional study of symptomatic deep venous thrombosis and pulmonary embolism in prostate cancer patients undergoing laparoscopic or robot-assisted laparoscopic radical prostatectomy. Eur Urol 2008;53:134-145.

25. Yee $\mathrm{CH}$, Chung V, Ip K, et al. Incidence of thromboembolism after robotic-assisted laparoscopic radical prostatectomy. J Endourol 2008;22:A40. 
26. Salomon L, Saint F, Anastasiadis AG, et al. Combined reporting of cancer control and functional results of radical prostatectomy. Eur Urol 2003;44:656-660.

27. Shikanov SA, Zorn KC, Zagaja GP, Shalhav AL. Trifecta outcomes after robotic-assisted laparoscopic prostatectomy. Urology 2009;74:619-623.

28. Patel VR, Coelho RF, Chauhan S, et al. Continence, potency and oncological outcomes after robotic-assisted radical prostatectomy: Early trifecta results of a high-volume surgeon. BJU Int 2010;106:696-702.

29. Ahlering TE, Skarecky D, Lee D, Clayman RV. Successful transfer of open surgical skills to a laparoscopic environment using a robotic interface: Initial experience with laparoscopic radical prostatectomy. J Urol 2003;170:1738-1741.

30. Murphy DG, Challacombe BJ, Costello AJ. Outcomes after robot-assisted laparoscopic radical prostatectomy. Asian J Androl 2009;11:94-99.

31. Denberg TD, Flanigan RC, Kim FJ, et al. Self-reported volume of radical prostatectomies among urologists in the USA. BJU Int 2007;99:339-343.

32. Barbash GI, Glied SA. New technology and health care coststhe case of robot-assisted surgery. N Engl J Med 2010;363: 701-704.
Address correspondence to: Chi-hang Yee, MBBS

Department of Surgery Prince of Wales Hospital

Shatin

Hong Kong

E-mail: yeechihang@surgery.cuhk.edu.hk 